\title{
Genetic variants at the miR-124 binding site on the cytoskeleton-organizing IQGAPI gene confer differential predisposition to breast cancer
}

\author{
HONG ZHENG $^{1,2^{*}}$, FENGJU SONG ${ }^{1,2^{*}}$, LINA ZHANG ${ }^{1,2}$, DA YANG ${ }^{3}$, PING JI ${ }^{3}$, YINGMEI WANG ${ }^{3}$, \\ MARIA ALMEIDA ${ }^{4}$, GEORGE A. CALIN ${ }^{4}$, XISHAN HAO ${ }^{1,2}$, QINGYI WEI ${ }^{5}$, WEI ZHANG ${ }^{3}$ and KEXIN CHEN $^{1,2}$ \\ ${ }^{1}$ Department of Epidemiology and Biostatistics, Tianjin Medical University Cancer Hospital and Institute; \\ ${ }^{2}$ Key Laboratory of Breast Cancer Prevention and Therapy, Tianjin Medical University, \\ Ministry of Education, Tianjin, P.R. China; Departments of ${ }^{3}$ Pathology, ${ }^{4}$ Experimental Therapeutics, \\ and ${ }^{5}$ Epidemiology, The University of Texas M.D. Anderson Cancer Center, Houston, TX, USA
}

Received October 20,2010; Accepted December 17, 2010

DOI: $10.3892 /$ ijo.2011.940

\begin{abstract}
IQGAPI knockout mice develop gastric cancer, but the IQGAP1 protein is associated with some advanced-stage human cancers. IQGAP1 expression is regulated by a microRNA, miR-124, through a binding site at the 3'-untranslated region, where a single nucleotide polymorphism (SNP) exists in the core binding region. We asked whether IQGAPI expression is associated with breast cancer development and whether genetic variants at the miR-124 binding site are important. We genotyped the IQGAPI SNP rs1042538 A/T in 1,541 breast cancer cases and 1,598 controls and analyzed the frequency of the variant and interactions with major risk factors in these populations. We also measured the expression of IQGAP1 at both mRNA and protein levels in different IQGAPI genotypes. The IQGAPI TT genotype, compared with the AA genotype, was associated with a significantly lower risk of developing breast cancer $[\mathrm{P}=0.049$, odds ratio $(\mathrm{OR}), 0.78 ; 95 \%$ confidence interval (CI), 0.61-0.99]. In case-only analyses, the TT, compared with the AA, genotype was associated with progesterone receptor-positive subjects (OR, 1.35; 95\% CI, 1.00-1.83). The expression levels of IQGAP1 protein were significantly higher in the TT genotype compated to the AA genotype. The presence of SNPs at the miR-124 binding site may be a marker for predicting breast cancer risk and prognosis.
\end{abstract}

Correspondence to: Dr Kexin Chen, Department of Epidemiology and Biostatistics, Tianjin Medical University Cancer Hospital and Institute, Tianjin 300060, P.R. China

E-mail: chenkexin1963@yahoo.com

Dr Wei Zhang, Department of Pathology, Unit 85, The University of Texas M.D. Anderson Cancer Center, 1515 Holcombe Blvd., Houston, TX 77030, USA

E-mail:wzhang@mdanderson.org

*Contributed equally

Key words: breast cancer, microRNA, SNP, IQGAP1, case-control study

\section{Introduction}

During embryonic development and throughout adult life in humans, gene expression is tightly regulated by a complex biological network in a tissue-specific manner and in close interaction with the environment to ensure accurate spatial and temporal differentiation of multiple organs with distinct functions. Significant deviation in the key regulators of this network via mutations often results in pathogenesis, including cancer. Subtle differences in the key regulatory genes via single nucleotide polymorphisms (SNPs) can result either in non-pathologic differences, such as hair color, or in increased propensity for a diseased state, such as cancer. Revealing the roles of these SNPs is a major area of research in the realm of molecular epidemiology in the era of postgenomic medicine (1).

In recent years, studies have revealed the importance of a class of small non-coding RNAs, microRNAs (miRNAs), that are critically involved in regulating gene expression (2). miRNAs directly regulate about $30 \%$ of the genes in the human genome via degradation or translational inhibition of their target messenger RNAs (mRNAs) and are thus important regulators of cellular processes such as differentiation, proliferation, mobility, and apoptosis $(3,4)$. The first miRNA-target mRNA pair to be verified in vivo was let-7 miRNA and its target, lin-41; and the natural interaction between this miRNA and its target is one of the best understood (5). miRNAs suppress gene expression mainly by binding to the complementary sequences in the 3 untranslated regions (UTRs) of mRNA of their target genes (6), although some miRNAs have been shown to act as an enhancer element to increase gene expression (7). It is generally believed that nucleotides $2-8$ from the 5 ' end of miRNA, called the 'seed region', are the most critical for miRNA binding (8). Perfect Watson-Crick complementarity is observed in these 7 consecutive base pairs in most cases. Therefore, a single nucleotide change in this region may cause sufficient disruption in binding to deregulate the target genes (9). If the target gene is an oncogene, a tumor suppressor gene, or other regulatory genes that are critical for homeostasis, the single nucleotide change in the miRNA binding site may alter 
the expression of these genes and shift the normal cellular program to a cancer-prone state, thus increasing cancer risk and/or conferring a specific cancer phenotype $(10,11)$.

Breast cancer is the most common cancer among women in developed countries, and an alarming increase in incidence has been seen in developing countries (12). Germline mutations in BRCA1 and BRCA2 genes account for only 5\% of all breast cancer cases in the general population $(13,14)$. Other low-penetrance genetic variants, especially in as-yet unknown combinations, are expected to explain most breast cancer incidence (15). Investigators, including those in our group, have hypothesized that the 3' UTRs of miRNA target genes may harbor such important variants $(10,16)$. Saunders et al (17) conducted a bioinformatic survey of the human genome for SNPs in putative miRNA target sites and found an appreciable level of variations within predicted as well as experimentally verified miRNA targets. One of the SNPs highlighted in the study of Saunders et al was SNP rs1042538 in the IQGAPI gene (encoding IQ motif-containing GTPase-activating protein 1), and this SNP has been experimentally verified to disrupt a miRNA target site sequence for miRNA-124 (miR-124) (18).

The scaffold protein IQGAP1 integrates signaling pathways and participates in diverse cellular activities that are important for both normal development and diseased states (19-24). IQGAP1 has attracted attention from cancer and developmental biologists because IQGAP1 expression appears to play an opposite role in normal development and in cancer progression. Studies with human tumor tissues have suggested that IQGAPI is an oncogene, which is overexpressed in a number of human solid neoplasms, including cancers of the colon, ovaries, stomach, and breast as well as glioblastoma (25-29). Functional studies have established the fact that IQGAP1 interacts with and regulates the actin-Cdc42/Rac1-mitogen-activated protein kinase pathway, thus contributing to its role in cell migration and invasion (19). Furthermore, another report suggested that IQGAP1 is involved in the expansion of cancer stem cells in glioblastoma and that together with IGFBP2 was associated with shorter survival in glioblastoma patients (27). Similarly, IQGAP1 was shown to be a marker of poor prognosis in ovarian cancer (26). In gastric cancer, it was shown that IQGAP1 was up-regulated by gene amplification (30).

However, the putative oncogenic properties of IQGAP1 have been contradicted by the finding that the deletion of IQGAPI in mice resulted in hyperplasia in gastric epithelial cells (31), suggesting that the IQGAPI gene plays an important role in normal development and actually has an antiproliferative function in normal epithelial cells. In other words, the IQGAPI gene may have a tumor suppression function in normal cells but may turn into an oncogene in tumor cells through an unknown mechanism. These conflicting properties of IQGAP1 suggest that IQGAP1 function may be dependent on developmental stage and cell type. However, determining the exact role and regulation of IQGAP1 in normal and cancer development will require more extensive studies, including population-based and functional studies.

A large number of SNPs have been found within the sequence of the IQGAPI gene, but SNP rs1042538 is recognized as the only one targeted by a miRNA with a high frequency of variation in Chinese population. This SNP (A/T variant) is one of the seven consecutive nucleotides corresponding to the seed region of miR-124 (Fig. 1). The A allele, together with the other six, form a perfect pairing with the seed, which is responsible for the down-regulation of IQGAP1 by miR-124 (17). Therefore, we conducted a case-control study to investigate whether this SNP at the miRNA-binding site on the IQGAP1 gene plays a role in breast cancer development and prognosis in a Chinese population.

\section{Patients and methods}

Patients and controls. Study patients were recruited from the Breast Cancer Research Center in Tianjin Medical University Cancer Hospital, and clinical information was acquired from the Tianjin Cancer Registry upon the approval of the Institutional Review Board (32). This study included 1,541 patients with newly diagnosed and histologically confirmed breast cancer, who were consecutively recruited between January 1, 2007 and February 28, 2008. The response rate of the eligible patients we recruited was $\sim 95 \%$.

We also recruited 1,598 cancer-free women (controls) during this study period who were genetically unrelated to one another and living in the nearby community. The response rate of the eligible controls who were approached for recruitment was $\sim 90 \%$. The controls were frequency-matched to the cases by age ( \pm 5 years).

After the study participants signed an informed consent form, they were interviewed for demographic data and information about major risk factors, including family history. For the cases, we also collected information about tumor features and disease severity, including morphologic characteristics, mean age at diagnosis, infiltrating/invasive ductal carcinoma (IDC) status, tumor size, presence of lymph node and/or organ metastasis, clinical stage, and estrogen receptor (ER) and progesterone receptor (PR) status. Each eligible subject donated $20 \mathrm{ml}$ of blood, which was collected into heparinized tubes and used for biomarker assays, including DNA extraction and genotyping.

Genotyping. From each blood sample, a leukocyte cell pellet, obtained from the buffy coat by centrifugation of $1 \mathrm{ml}$ of whole blood, was used for DNA extraction. Genomic DNA was isolated with the Qiagen DNA Blood Mini Kit (Qiagen Inc., Valencia, CA) according to the manufacturer's instructions. For genotyping the rs $1042538 \mathrm{~A} / \mathrm{T}$ SNP, both the amplifying primers and the TaqMan MGB probes were designed for the TaqMan SNP Genotyping assays (Applied Biosystems, Foster City, CA). More than $10 \%$ of the samples were randomly selected for repeated assays, and the results were $100 \%$ concordant.

Quantitative measurement of IQGAPI $m R N A$ and protein expression. Total RNA was isolated from 37 frozen breast cancer tissues from patients with known genotypes of IQGAPI (AA or TT), as determined from their blood samples. The extraction and purification of total RNA were performed with TRIzol reagent (Invitrogen) according to the manufacturer's instructions. RNA quality and concentration were determined with the Agilent 2100 Bioanalyzer (Agilent Technologies). Real-time quantitative polymerase chain reaction (PCR) was performed in a 96-well reaction plate (MicroAmp ${ }^{\circledR}$ Optical 

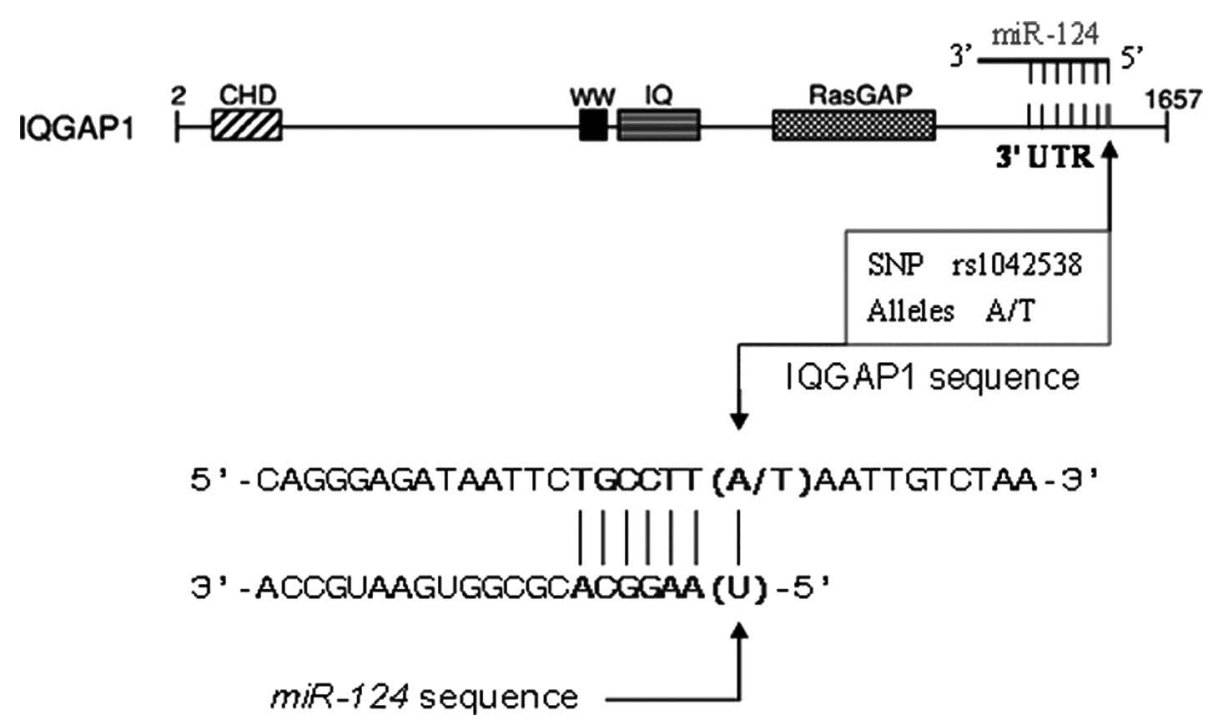

Figure 1. The base-pairing of the miR-124 seed region and the 3'-UTR of the IQGAP1 gene, including the rs1042538 polymorphism. SNP rs1042538 has an $\mathrm{A} / \mathrm{T}$ variation, which is one of the seven consecutive nucleotides corresponding to the seed region of miR-124. The A allele, together with the other six, forms a perfect pairing with the seed, which is responsible for the down-regulation of IQGAP1 by miR-124; the T allele forms a non-perfect pairing with the seed and may escape the regulation of miR-124.

96-Well Reaction Plate, Applied Biosystems) on an ABI PRISM ${ }^{\circledR}$ 7500 Sequence Detector System (Applied Biosystems), according to the manufacturer's instructions.

RT-PCR for IQGAP1 expression was done using power SYBR Green one-step RT-PCR master mix reagent kit (P/N 4391178). All primers were synthesized by Sangon Corp. (Shanghai, China). In order to normalize the differences in the amount of total RNA used in each reaction, glyceraldehyde-3phosphate dehydrogenase (GAPDH) RNA was measured as endogenous control. Each sample was analyzed in duplicate and the coefficient of variation of all reactions was $<5 \%$. The relative expression level of IQGAP1 to GADPH was described using the equation: expression $=2^{-\Delta C t}$. Western blotting was done to evaluate the effect of miR-124 on the expression of IQGAP1 in cell lines, MM231 and LN299, using actin as a control. IQGAP1 protein expression was measured through Western blotting using actin as a loading control in 48 breast cancer tissues with IQGAP1 AA or TT genotype.

Statistical analyses. We used the $\chi^{2}$ test to compare differences in frequency distributions of demographic variables, risk factors, and alleles of the IQGAPI polymorphism between the cases and controls. We also tested the Hardy-Weinberg equilibrium of genotype distributions in the controls. In addition, we used unconditional univariate and multivariate logistic regression analyses to examine the association between the SNP and breast cancer risk by estimating odds ratios (ORs) and 95\% confidence intervals (CIs) with and without adjustment for age and other risk factors. Finally, we stratified the genotype data according to age, family history, and clinical variables (including morphologic characteristics, tumor size, presence of lymph node and/or organ metastases, tumor stage, and ER and PR status) of breast cancer patients by using the $\chi^{2}$ test and logistic regression. The expression levels of IQGAP1 between AA and TT genotypes were compared with use of the rank-sum test. All statistical analyses were two-sided and performed with use of SAS software (version 9.0; SAS Institute, Cary, $\mathrm{NC}$ ), and a $\mathrm{P}=0.05$ was considered statistically significant.

\section{Results}

Case-control analysis of the IQGAPI SNP. This case-control study included 1,541 breast cancer cases and 1,598 controls, and the distributions of known risk factors between cases and controls are shown in Table I. Age was adequately matched between cases and controls $(\mathrm{P}=0.242)$.

Genomic DNA for IQGAPI SNP genotyping was isolated from the peripheral blood of all participants with use of the TaqMan assay. The distribution of the IQGAPI genotypes (rs 1042538) is shown in Table II. The SNP was in HardyWeinberg equilibrium in controls $(\mathrm{P}=0.469)$. The AA genotype was found in $33.42 \%$ of cases and $32.17 \%$ of controls, whereas the TT genotype was found in $17.26 \%$ of cases and $19.52 \%$ of controls. The TT genotype, compared with the AA genotype, was associated with a significantly lower risk of developing breast cancer ( $\mathrm{P}=0.049$, OR, 0.78; 95\% CI, 0.61-0.99). In other words, women with the AA genotype were more likely than those with the TT genotype to develop breast cancer.

We also separated the cases into those with IDC (about 2/3 of all cases) and others (mainly including adenocarcinoma, carcinoma simplex, mucinous carcinoma, medullary carcinoma, lobular carcinoma). The two groups of cases were used in case-control analyses, and results revealed that in non-IDC cases only, those with the TT genotype had a significantly lower risk of developing cancer (OR=0.69; 95\% CI, 0.50-0.94) than did those with the AA genotype (Table II). Thus, it appears that the effect of the IQGAP1 SNP is less apparent in more aggressive and invasive stages of breast cancer, when many genetic and epigenetic factors become involved.

Case-only analysis of IQGAPI genotypes. The patient data we collected included information on mean age at diagnosis, 
Table I. Frequency distributions of selected variables in breast cancer cases and cancer-free controls in Chinese women.

No. (\%) of subjects

Variables

Cases $(\mathrm{n}=1,541) \quad$ Controls $(\mathrm{n}=1,598)$

OR $(95 \% \mathrm{CI})$

P-value

Age (years)

$\leq 50$

$>50$

739 (47.96)

$733(45.87)$

$865(54.13)$

Frequency of pregnancy

$\leq 2$

$710(46.07)$

$728(45.56)$

1.00

0.771

$>2$

831 (53.93)

$870(54.44)$

$0.94(0.80-1.11)$

Duration of breastfeeding (months)

$\begin{array}{ll}\leq 12 & 733(47.57) \\ >12 & 808(52.43)\end{array}$

$731(47.65)$

$803(52.35)$

$706(44.54)$

879 (55.46)

1.00

$0.81(0.64-1.03)$

Oral contraception ${ }^{\mathrm{c}}$

Never

1,197 (81.87)

$265(18.13)$

1,321 (84.46)

243 (15.54)

1.00

0.057

Ever

Smoking status ${ }^{\mathrm{c}}$

Never

$1,309(87.85)$

181 (12.15)

$1,478(93.25)$

$107 \quad(6.75)$

1.00

$<0.001$

Ever

1,129 (73.79)

1,465 (92.78)

$114 \quad(7.22)$

1.00

$<0.001$

401 (26.21)

$4.41(3.49-5.58)$

$2.23(1.70-2.93)$

Benign breast disease ${ }^{c}$

Ever

$$
\begin{array}{r}
1,061(68.90) \\
479(31.10)
\end{array}
$$

1.00

$<0.001$
Yes

CI, confidence interval; OR, odds ratio. ${ }^{a}$ Two-sided $\chi^{2}$ test. ${ }^{b}$ First- and second-degree relatives. ${ }^{c}$ Due to missing values, the number of cases and controls are less than 1,541 and 1,598 , respectively.

IDC status, tumor size, presence of lymph node and/or organ metastasis, clinical stage, and ER/PR status. Our analysis did not reveal association between any genotypes and mean age at diagnosis ( $\mathrm{P}=0.387$ ), IDC status (IDC vs. non-IDC, $\mathrm{P}=0.194$ ), tumor size $(\mathrm{P}=0.821)$, lymph node metastases $(\mathrm{P}=0.652)$, clinical stage $(\mathrm{P}=0.773)$, or ER status $(\mathrm{P}=0.386)$ (Table IV). Our analysis revealed significant association between genotypes and PR status of cases. Specifically, cases with the TT genotype were more likely to be PR-positive than were those with the AA genotype (OR, 1.35; 95\% CI, 1.00-1.83) (Table III). Furthermore, in ER-negative cases, the IQGAP1 genotype was significantly associated with PR status. Cases with AT or TT genotypes, compared with the AA genotype, were more likely to be
PR-positive ( $\mathrm{P}=0.022)$ (OR for AT genotype, 1.50; 95\% CI, 1.01-2.23; OR for TT genotype, 1.91; 95\% CI, 1.18-3.09) (Table IV).

Analysis of IQGAPI expression. Our hypothesis was that IQGAP1 TT genotype, evading the regulation of miR-124, would have a higher level of IQGAP1 expression. We thus measured IQGAP1 expression at mRNA and protein levels using breast cancer tissue. IQGAP1 mRNA was higher in AA genotype than in TT genotype ( 0.040 vs. 0.025$)$, the difference between the AA genotype and the TT genotype was not significant (rank-sum test, $\mathrm{P}=0.109$ ) (Fig. 2A). The relative IQGAP1 protein levels were higher in TT genotype than in 
AA genotype (125.46 vs. 51.35), the difference between the TT genotype and the AA genotype was statistically significant (rank sum test, $\mathrm{P}=0.039$ ) (Fig. 2B).

\section{Discussion}

We began this case-control study to find an answer to the question of whether the SNP at the miR-124 binding site on the 3'-UTR of the cytoskeleton-organizing gene IQGAPI had any effect on breast cancer risk. We did not have an a prior prediction because the published results on the function of IQGAP1 appeared to be contradictory. On the one hand, removal of the IQGAPI gene predisposed mice to the development of gastric cancer. On the other hand, IQGAP1 expression was elevated in many cancers and in some tumors correlated with poor prognosis. Our case-control study showed that the AA genotype of the miR-124 binding site SNP on IQGAP I, which renders its down-regulation by miR-124, was associated with increased risk of breast cancer. Although this does not prove that IQGAP1 plays a role in tumor suppression, the result is consistent with the knockout mouse studies that suggest that IQGAP1 is a negative regulator for cancer development. We also examined IQGAP1 expression in breast cancer tissues and their adjacent pathologically normal tissues and found that indeed, IQGAP1 was up-regulated in breast cancer tissue, similar to findings in other previously reported studies (33). Moreover, IQGAP1 knockdown experiments showed that reduction of IQGAP1 resulted in increased cell growth in non-cancer breast epithelial cells but resulted in decreased cell growth in breast cancer cells, supporting that IQGAP1 plays an opposite role in normal development and cancer. Or in other words, IQGAP1 may indeed act as a tumor suppressor in breast cancer initiation but act as an oncogene after cancer has developed. Therefore, our findings may help explain the apparently contradictory results regarding IQGAP1 in the same human populations, at least partially removing the argument that IQGAP1 may work differently in humans and mice.

The A allele, which binds perfectly with miR-124 and leads to the down-regulation of IQGAP1 expression, is associated with increased risk of breast cancer. This finding suggests that IQGAP1, and by extension cytoskeleton organization, is critical for normal breast cell development. A lack of IQGAP1 and disrupted cytoskeleton organization contributed to breast cancer development or hyperplasia in gastric epithelial cells in the mouse studies (31), although it is not known whether there were any abnormalities in breast epithelial cells in the IQGAPI gene knockout mice or whether the A allele of our studied SNP had any effect on gastric cancer development. Nevertheless, because cytoskeleton structure is critical for maintaining highly organized and polarized epithelia cell sheets, the requirement for sufficient IQGAP1 expression is quite logical. Further case-control studies are clearly warranted in other types of cancer.

Our population-based case-control study suggests that the $\mathrm{T}$ allele of the miR-124 binding site SNP on the IQGAP1 gene is a 'good' variant that has a breast cancer protection function. Of interest, the $\mathrm{T}$ allele was found in frequencies of 40,10 , and $10 \%$, respectively, for the HapMap panels of Asian, European, and African women. It is well established that breast cancer 
Table III. Association of the IQGAPI polymorphism with clinical characteristics of breast cancer cases.

\begin{tabular}{|c|c|c|c|c|c|}
\hline \multirow[b]{2}{*}{ Variables } & \multirow[b]{2}{*}{$\begin{array}{l}\text { No. of } \\
\text { patients }\end{array}$} & \multicolumn{3}{|c|}{ IQGAP1 rs1042538 (A/T) genotype frequency } & \multirow[b]{2}{*}{ P-value ${ }^{a}$} \\
\hline & & $\begin{array}{c}\mathrm{AA} \\
(\mathrm{n}=515)\end{array}$ & $\begin{array}{c}\mathrm{AT} \\
(\mathrm{n}=760)\end{array}$ & $\begin{array}{c}\text { TT } \\
(\mathrm{n}=266)\end{array}$ & \\
\hline Age at diagnosis (years) & $1,540^{\mathrm{b}}$ & $51.61 \pm 10.50$ & $52.13 \pm 11.11$ & $51.14 \pm 10.18$ & 0.387 \\
\hline Morphology & 1,541 & & & & \\
\hline IDC & & $345(66.99)$ & $525(69.08)$ & $195(73.31)$ & 0.194 \\
\hline Others & & $170(33.01)$ & 235 (30.92) & $71(26.69)$ & \\
\hline OR $(95 \% \mathrm{CI})$ & & 1.00 & $0.91(0.72-1.15)$ & $0.74(0.53-1.03)$ & \\
\hline Tumor size & $1,395^{\mathrm{b}}$ & & & & \\
\hline$\leq 2 \mathrm{~cm}$ & & $171(36.93)$ & $242(35.12)$ & $87(35.80)$ & 0.821 \\
\hline$>2 \mathrm{~cm}$ & & $292(63.07)$ & $447(64.88)$ & $156(64.20)$ & \\
\hline OR $(95 \% \mathrm{CI})$ & & 1.00 & $1.08(0.85-1.38)$ & $1.05(0.76-1.45)$ & \\
\hline Lymph node metastases & $1,534^{\mathrm{b}}$ & & & & \\
\hline No & & $309(60.47)$ & $473(62.48)$ & 159 (59.77) & 0.652 \\
\hline Yes & & $202(39.53)$ & $284(37.52)$ & $107(40.23)$ & \\
\hline OR $(95 \% \mathrm{CI})$ & & 1.00 & $0.92(0.73-1.16)$ & $1.03(0.76-1.39)$ & \\
\hline Clinical stage & $1,394^{\mathrm{b}}$ & & & & \\
\hline $0+\mathrm{I}$ & & $122(26.41)$ & 193 (27.97) & $63(26.03)$ & 0.773 \\
\hline II+III+IV & & $340(73.59)$ & 497 (72.03) & $179(73.97)$ & \\
\hline OR $(95 \% \mathrm{CI})$ & & 1.00 & $0.92(0.71-1.21)$ & $1.02(0.72-1.45)$ & \\
\hline ER & $1,515^{\mathrm{b}}$ & & & & \\
\hline- & & $224(44.09)$ & $314(42.32)$ & 125 (47.17) & 0.386 \\
\hline+ & & $284(55.91)$ & $428(57.68)$ & $140(52.83)$ & \\
\hline OR $(95 \% \mathrm{CI})$ & & 1.00 & $1.08(0.86-1.35)$ & $0.88(0.66-1.19)$ & \\
\hline PR & $1,515^{\mathrm{b}}$ & & & & \\
\hline- & & $241(47.44)$ & $314(42.32)$ & $106(40.00)$ & 0.085 \\
\hline+ & & 267 (52.56) & $428(57.68)$ & $159(60.00)$ & \\
\hline OR $(95 \% \mathrm{CI})$ & & 1.00 & $1.23(0.98-1.54)$ & $1.35(1.00-1.83)^{c}$ & \\
\hline
\end{tabular}

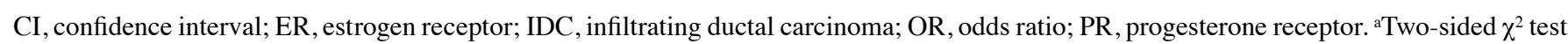
for difference in frequency distribution of variables between IQGAPI genotypes. b Due to missing values, n is $<1,541$. ${ }^{c}$ Statistically significant.

incidence is much lower among Asian women than among women of European or African descent. Such differences have often been attributed to different lifestyles in the absence of known genetic factors. However, if the $\mathrm{T}$ allele is indeed protective, this allele and other similar alleles may, at least in part, constitute the genetic basis for the varying incidence in breast cancer among Asian, European, and African women. Future population-based studies in European and African women cohorts will be needed to test this hypothesis.

In our study, we also evaluated the potential association of this SNP with breast cancer prognosis. This was because
IQGAP1 has been shown to be overly expressed in cancer, and expression levels have been associated with poor prognosis in several other cancer types, including glioblastoma (34). This association is not surprising because enhanced cell migration, invasion, and metastasis of cancer cells require heightened cytoskeleton reorganizing activities. However, we were initially surprised with our analysis results that showed a lack of correlation of this SNP with major clinical parameters such as metastasis and tumor invasiveness in our breast cancer cases. We did observe some association with PR status, but the significance of this association is currently unclear. A few 
Table IV. Association of the IQGAP1 polymorphism with clinical characteristics of breast cancer in estrogen receptor-negative cases.

\begin{tabular}{|c|c|c|c|c|c|}
\hline \multirow[b]{2}{*}{ Variables } & \multirow[b]{2}{*}{$\begin{array}{l}\text { No. of } \\
\text { patients }\end{array}$} & \multicolumn{3}{|c|}{ IQGAP1 rs1042538 (A/T) genotype frequency } & \multirow[b]{2}{*}{ P-value ${ }^{a}$} \\
\hline & & $\begin{array}{c}\mathrm{AA} \\
(\mathrm{n}=224)\end{array}$ & $\begin{array}{c}\text { AT } \\
(\mathrm{n}=314)\end{array}$ & $\begin{array}{c}\mathrm{TT} \\
(\mathrm{n}=125)\end{array}$ & \\
\hline Tumor size & $603^{\mathrm{b}}$ & & & & \\
\hline$\leq 2 \mathrm{~cm}$ & & $64(32.00)$ & $97(33.68)$ & $36(31.30)$ & 0.873 \\
\hline$>2 \mathrm{~cm}$ & & $136(68.00)$ & $191(66.32)$ & $79(68.70)$ & \\
\hline OR $(95 \% \mathrm{CI})$ & & 1.00 & $0.93(0.63-1.36)$ & $1.03(0.63-1.69)$ & \\
\hline Lymph node metastases & 663 & & & & \\
\hline No & & $129(57.59)$ & 199 (63.38) & $78(62.40)$ & 0.381 \\
\hline Yes & & $95(42.41)$ & 115 (36.62) & $47(37.60)$ & \\
\hline OR $(95 \% \mathrm{CI})$ & & 1.00 & $0.79(0.55-1.11)$ & $0.82(0.52-1.28)$ & \\
\hline Clinical stage & $603^{\mathrm{b}}$ & & & & \\
\hline $0+\mathrm{I}$ & & $41(20.60)$ & $74(25.61)$ & $27(23.48)$ & 0.441 \\
\hline II+III+IV & & $158(79.40)$ & $215(74.39)$ & $88(76.52)$ & \\
\hline OR $(95 \% \mathrm{CI})$ & & 1.00 & $0.75(0.49-1.16)$ & $0.85(0.48-1.47)$ & \\
\hline Morphology & 663 & & & & \\
\hline IDC & & $158(70.54)$ & $223(71.02)$ & $93(74.40)$ & 0.721 \\
\hline Others & & $66(29.46)$ & $91(28.98)$ & $32(25.60)$ & \\
\hline OR $(95 \% \mathrm{CI})$ & & 1.00 & $0.98(0.67-1.42)$ & $0.82(0.50-1.35)$ & \\
\hline PR & $662^{\mathrm{b}}$ & & & & \\
\hline- & & $173(74.76)$ & $217(70.21)$ & $80(63.30)$ & 0.022 \\
\hline+ & & $51(25.24)$ & $96(29.79)$ & 45 (36.70) & \\
\hline OR $(95 \% \mathrm{CI})$ & & 1.00 & $1.50(1.01-2.23)^{\mathrm{c}}$ & $1.91(1.18,3.09)^{\mathrm{c}}$ & \\
\hline
\end{tabular}

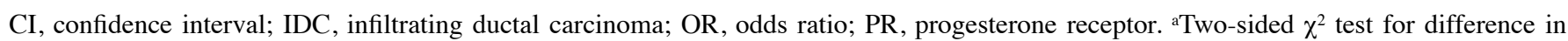

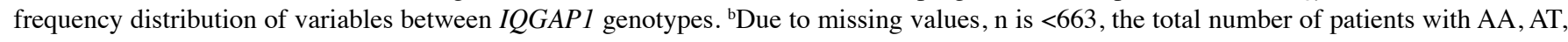
or TT genotypes. 'Statistically significant.
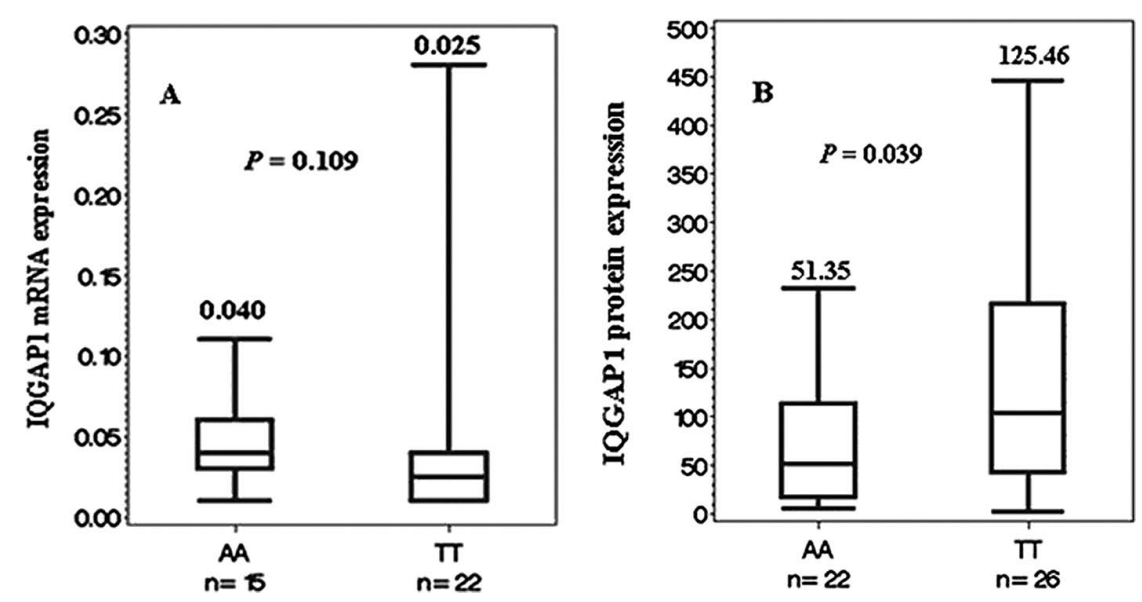

Figure 2. IQGAP1 expression in tumor tissue with IQGAP1 AA or TT genotypes. (A) Comparison of IQGAP1 expression at mRNA level between IQGAP1 genotypes (AA and TT) in breast cancer tissue. The difference of IQGAP1 mRNA between the TT genotype and the AA genotype was not significant (rank-sum test, $\mathrm{P}=0.109)$. (B) Comparison of IQGAP1 expression at protein level between IQGAP1 genotypes (AA and TT) in breast cancer tissue. IQGAP1 protein was higher in TT genotype than in AA genotype, the difference between the TT genotype and the AA genotype was statistically significant (rank-sum test, $\mathrm{P}=0.039)$. 
other studies have also shown an association between genetic variations and PR status. In a case-control study with more than 9,000 subjects, Pooley et al (35) found that a coding SNP (rs3218536) in one of the DNA double-strand break repair genes, $X R C C 2$, was strongly associated with risk of developing PR-positive breast cancer. In another study, Synowiec et al (36) reported an association between the $R A D 51-135 \mathrm{G} / \mathrm{C}$ polymorphism and $\mathrm{PR}$ expression $(\mathrm{OR}=6.33$; $95 \% \mathrm{CI}=1.15-35.01)$.

Our results suggest a number of possibilities. First, IQGAP1 expression in breast cancer may not be strongly regulated by miR-124, and thus the SNP site for miR-124 binding may be irrelevant. Although we do not have direct evidence to support this possibility, one study found that miR-124 promoter is methylated in acute lymphoblastic leukemia (ALL) and that the expression of miR-124 in ALL is very low (37). Our analysis of miR-124 expression supported this observation that miR-124 is decreased in cancer tissue compared with adjacent normal tissue. A second scenario is that IQGAP1 expression levels may be less important to breast cancer because other genes in the same pathway are more activated. If this is true, then the expression of IQGAP1 should not be correlated with prognosis of breast cancer. We tested this hypothesis by examining the gene expression profile data in the public domain and showed that indeed, IQGAP1 expression levels in two breast cancer datasets did not correlate with grade or metastasis. We also showed that IQGAP1 expression did not correlate with grade in gastric cancer. In contrast, IQGAP1 expression levels were associated with grade and poor survival in gliomas, consistent with the literature.

In this study, we could not evaluate the association between IQGAP1 SNP genotypes and breast cancer survival, mainly because the breast cancer cases recruited for our study were relatively recent, which meant that there was not enough follow-up time for analysis of survival or distant metastasis. However, in the van't Veer et al (38) study, clinical follow-up of 78 breast cancer patients for more than 5 years after lumpectomy showed that IQGAP1 expression levels were not correlated with distance metastasis. Therefore, the role of IQGAP1 in cancer prognosis is apparently dependent on cancer type.

In conclusion, we have conducted the first epidemiologic study of the association between the IQGAPI SNP within the miR124 binding site and breast cancer risk. Our study showed that the regulation of IQGAP1 could be complex and that the role of IQGAP1 in cancer cannot be generalized. However, the role of this SNP in cancer risk may be broader because of the different frequency distributions of demographic variables, risk factors, and alleles of the IQGAP1 polymorphism between Chinese women and women of other ethnic groups. Because of its potential significance, this study should be replicated with populations of various ethnic backgrounds. Once validated, this SNP may be important for genetic testing and screening of individuals at high risk of breast cancer.

\section{Acknowledgments}

We thank Hongwei Han, Lei Lei, and Yanrui Zhao for their technical assistance. We also thank Ms. Tamara Locke of the Department of Scientific Publications at M.D. Anderson
Cancer Center for editing this manuscript. This work was supported partially by grants from the National Natural Science Foundation of China (No. 30872172; No. 30771844), the Tianjin Science and Technology Committee Foundation (No.08ZCGHZ02000;09ZCZDSF04700 and08JCZDJC23600), Important National Science and Technology Specific Projects (2011ZX09307-001-04) and the Major State Basic Research Development Program of China (973 Program) (No. 2009CB918903). The tissue bank is jointly supported by the Tianjin Cancer Institute and Hospital and National Foundation for Cancer Research (US).

\section{References}

1. Zhang C: MicroRNomics: a newly emerging approach for disease biology. Physiol Genomics 33: 139-147, 2008.

2. Kim VN: Small RNAs: classification, biogenesis, and function. Mol Cells 19: 1-15, 2005.

3. Tsuchiya S, Okuno Y and Tsujimoto G: MicroRNA: biogenetic and functional mechanisms and involvements in cell differentiation and cancer. J Pharmacol Sci 101: 267-270, 2006.

4. Wiemer EA: The role of microRNAs in cancer: no small matter. Eur J Cancer 43: 1529-1544, 2007.

5. Vella MC, Reinert K and Slack FJ: Architecture of a validated microRNA:target interaction. Chem Biol 11: 1619-1623, 2004.

6. Evans SC, Kourtidis A, Markham TS, Miller J, Conklin DS and Torres AS: MicroRNA target detection and analysis for genes related to breast cancer using MDLcompress. EURASIP J Bioinform Syst Biol: 43670, 2007.

7. Vasudevan S, Tong Y and Steitz JA: Switching from repression to activation: microRNAs can up-regulate translation. Science 318: 1931-1934, 2007.

8. Shivdasani RA: MicroRNAs: regulators of gene expression and cell differentiation. Blood 108: 3646-3653, 2006.

9. Yu Z, Li Z, Jolicoeur N, et al: Aberrant allele frequencies of the SNPs located in microRNA target sites are potentially associated with human cancers. Nucleic Acids Res 35: 4535-4541, 2007.

10. Landi D, Gemignani F, Barale R and Landi S: A catalog of polymorphisms falling in microRNA-binding regions of cancer genes. DNA Cell Biol 27: 35-43, 2008.

11. Song F, Zheng H, Liu B, et al: An miR-502-binding site singlenucleotide polymorphism in the 3'-untranslated region of the SET8 gene is associated with early age of breast cancer onset. Clin Cancer Res 15: 6292-6300, 2009.

12. Jemal A, Siegel R, Ward E, Hao Y, Xu J and Thun MJ: Cancer statistics, 2009. CA Cancer J Clin 59: 225-249, 2009.

13. Chen YC and Hunter DJ: Molecular epidemiology of cancer. CA Cancer J Clin 55: 45-57, 2005.

14. McPherson K, Steel CM and Dixon JM: ABC of breast diseases. Breast cancer-epidemiology, risk factors, and genetics. BMJ 321: 624-628, 2000

15. Pharoah PD, Tyrer J, Dunning AM, Easton DF and Ponder BA: Association between common variation in 120 candidate genes and breast cancer risk. PLoS Genet 3: e42, 2007.

16. Chen K, Song F, Calin G A, Wei Q, Hao X and Zhang W: Polymorphisms in microRNA targets: a gold mine for molecular epidemiology. Carcinogenesis 29: 1306-1311, 2008.

17. Saunders MA, Liang H and Li WH: Human polymorphism at microRNAs and microRNA target sites. Proc Natl Acad Sci USA 104: 3300-3305, 2007.

18. Lim LP, Lau NC, Garrett EP, et al: Microarray analysis shows that some microRNAs downregulate large numbers of target mRNAs. Nature 433: 769-773, 2005.

19. Noritake J, Watanabe T, Sato K, Wang S and Kaibuchi K: IQGAP1: a key regulator of adhesion and migration. J Cell Sci 118: 2085-2092, 2005.

20. Meyer RD, Sacks DB and Rahimi N: IQGAP1-dependent signaling pathway regulates endothelial cell proliferation and angiogenesis. PLoS One 3: e3848, 2008.

21. Wang Y, Wang A, Wang F, et al: IQGAP1 activates Tcf signal independent of Racl and Cdc42 in injury and repair of bronchial epithelial cells. Exp Mol Pathol 85: 122-128, 2008.

22. Ren JG, Li Z and Sacks DB: IQGAP1 integrates $\mathrm{Ca}^{2+} /$ calmodulin and B-Raf signaling. J Biol Chem 283: 22972-22982, 2008. 
23. Wang $\mathrm{Y}$, Wang $\mathrm{M}$, Wang $\mathrm{F}$, et al: IQGAP1 promotes cell proliferation and is involved in a phosphorylation-dependent manner in wound closure of bronchial epithelial cells. Int J Mol Med 22: 79-87, 2008

24. Mataraza JM, Briggs MW, Li Z, Entwistle A, Ridley AJ and Sacks DB: IQGAP1 promotes cell motility and invasion. J Biol Chem 278: 41237-41245, 2003.

25. Nabeshima K, Shimao $Y$, Inoue $T$ and Koono M: Immunohistochemical analysis of IQGAP1 expression in human colorecta carcinomas: its overexpression in carcinomas and association with invasion fronts. Cancer Lett 176: 101-109, 2002.

26. Dong P, Nabeshima K, Nishimura N, et al: Overexpression and diffuse expression pattern of IQGAP1 at invasion fronts are independent prognostic parameters in ovarian carcinomas. Cancer Lett 243: 120-127, 2006.

27. Balenci L, Clarke ID, Dirks PB, et al: IQGAP1 protein specifies amplifying cancer cells in glioblastoma multiforme. Cancer Res 66: 9074-9082, 2006

28. Johnson M, Sharma M and Henderson BR: IQGAP1 regulation and roles in cancer. Cell Signal (In press).

29. Nakamura H, Fujita K, Nakagawa H, et al: Expression pattern of the scaffold protein IQGAP1 in lung cancer. Oncol Rep 13: 427-431, 2005

30. Walch A, Seidl S, Hermannstadter C, et al: Combined analysis of Rac1, IQGAP1, Tiam1 and E-cadherin expression in gastric cancer. Mod Pathol 21: 544-552, 2008.
31. Li S, Wang Q, Chakladar A, Bronson RT and Bernards A Gastric hyperplasia in mice lacking the putative $\mathrm{Cdc} 42$ effector IQGAP1. Mol Cell Biol 20: 697-701, 2000.

32. Song F, He M, Li H, et al: A cancer incidence survey in Tianjin: the third largest city in China - between 1981 and 2000. Cancer Causes Control 19: 443-450, 2008.

33. Jadeski L, Mataraza JM, Jeong HW, Li Z and Sacks DB: IQGAP1 stimulates proliferation and enhances tumorigenesis of human breast epithelial cells. J Biol Chem 283: 1008-1017, 2008.

34. McDonald KL, O'Sullivan MG, Parkinson JF, et al: IQGAP1 and IGFBP2: valuable biomarkers for determining prognosis in glioma patients. J Neuropathol Exp Neurol 66: 405-417, 2007.

35. Pooley KA, Baynes C, Driver KE, et al: Common singlenucleotide polymorphisms in DNA double-strand break repair genes and breast cancer risk. Cancer Epidemiol Biomarkers Prev 17: 3482-3489, 2008.

36. Synowiec E, Stefanska J, Morawiec Z, Blasiak J and Wozniak K: Association between DNA damage, DNA repair genes variability and clinical characteristics in breast cancer patients. Mutat Res 648: 65-72, 2008.

37. Agirre X, Vilas ZA, Jimenez VA, et al: Epigenetic silencing of the tumor suppressor microRNA Hsa-miR-124a regulates CDK6 expression and confers a poor prognosis in acute lymphoblastic leukemia. Cancer Res 69: 4443-4453, 2009.

38. van't Veer LJ, Dai H, et al: Gene expression profiling predicts clinical outcome of breast cancer. Nature 415: 530-536, 2002 